\title{
COMPUTER PROGRAMS FOR CALCULATING \\ ATOMIC DATA FOR IONS
}

\author{
W. EISSNER \\ Dept. of Physics, University College London, England
}

\begin{abstract}
This report is a continuation of the preceding review talk. I shall describe general purpose computer programs that have been developed in Professor Seaton's group at University College London. Other speakers will present results produced with them.

The programs require a minimum of input. General purpose means; not restricted to one ion or just one isoelectronic sequence. High speed is achieved by using approximations suitable for medium and highly ionized light atoms. The programs allow fully for configuration mixing, which plays an important role in ion calculations. Equations in M.J.Seaton's review will be referred to as (S.number).
\end{abstract}

\section{Atomic Structure Program}

This program deals with the problems of Sections 1 and 2 of Seaton's preceding talk. It uses non-relativistic wave functions based on a scaled statistical model potential. Therefore, it may be applied to cases with not fewer than 3 or 4 electrons, $N \gtrsim 3$, and elements not too far beyond $\mathrm{Fe}, Z=26$. It has been described in 1969 by Eissner and Nussbaumer. Jones (1970) has extended the program by including relativistic effects and fine structure. Eissner and Jones (1970) have outlined how the radiative transition problem is treated.

The program consists of two primary branches, and each requires a short string of input data:

$$
\begin{aligned}
& \text { (1) }[N:] C_{1}, C_{2}, \ldots C_{M} \\
& \text { (2) } Z ; \mathscr{M} ; \lambda_{s}^{0}, \lambda_{p}^{0}, \ldots \sigma_{1 s}, \sigma_{2 s}, \sigma_{2 p}, \ldots \\
& \hline
\end{aligned}
$$

The first branch, which deals with the purely algebraic angular problems, is supplied with a list of all configurations $C_{m}$ to be included in an $N$ electron atom. Thus the input

$$
C_{1}=1 s^{2} 2 s^{2}, \quad C_{2}=1 s^{2} 2 p^{2}
$$

for the Be sequence would treat the four lowest terms $S_{i} L_{i}$ with even parity $p-$ and take account of the important interaction between the two terms ${ }^{1} S$.

The second branch requires the atomic number $Z$; one may recycle through it, and re-use the algebraic data from the first branch for a series of isoelectronic ions. Term energies $E_{i}=E\left(S_{i} L_{i}\right)$ are computed by varying scaling parameters $\lambda_{l}$ in the statistical model potential (S.11), until the sum over the $\mathscr{M}$ lowest terms is a minimum:

$$
\sum_{i=1}^{\mathscr{H}} g_{i} E_{i}=\operatorname{Min}
$$

where $g_{i}=\left(2 S_{i}+1\right)\left(2 L_{i}+1\right)$ is the statistical weight. 
If the input data following $M$ are omitted, computation is started with a standard initial value for the $\lambda$ 's and the screening factors $\sigma_{n l}$ (which describe how much $Z$ is screened by the other $N-1$ electrons). Execution time may then increase. When recycling one can use the previous results as starting values. The $\lambda$ 's are close to unity.

Schematically the output reads

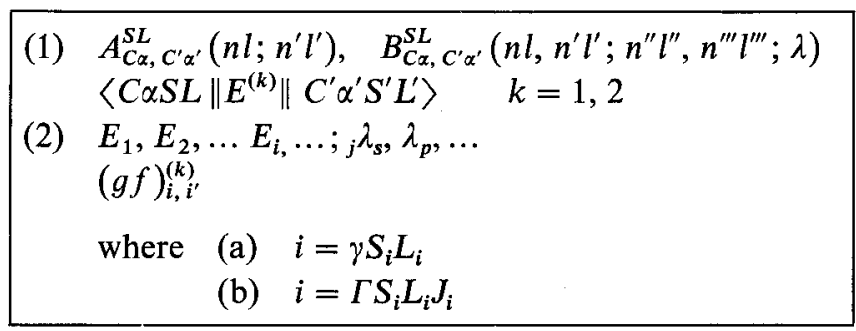

The first line of algebraic data (1) can be used as input to more refined methods (multi-configuration Hartree-Fock). The second line are reduced elements for electric dipole and quadrupole radiation. In (2) the extended version (b) of the program gives fine structure data as well. A fairly simple extension will calculate magnetic dipole transitions in our non-relativistic approximation. As a pure matter of notation: as long as there is a dominant configuration to a term, $\gamma$ or $\Gamma$ can be represented by a $C$. Typical execution time: 3 minutes on a CDC 6600 computer for one run with half a dozen electrons and configurations.

\section{Electron Collision Program}

This program computes data for exciting terms $S_{i} L_{i}$ of ions by electron impact in a partial wave method. It uses a distorted wave approximation; the radial functions of the colliding electrons, for $\varphi_{A}$ in (S. 15), are calculated in a statistical model potential. As target functions $\Psi_{A}$ in (S. 17), statistical model bound state functions are used: they are reconstructed from scaling parameters $\lambda_{l}$ as input, obtained by the Atomic Structure Program (1).

Again the program is fully automatic, requiring as input only

\begin{tabular}{|ll|}
\hline$(1.1)$ & {$[N:] C_{1}, C_{2}, C_{3} \ldots$} \\
$(1.2)$ & $(S L p)_{1}^{T},(S L p)_{2}^{T},(S L p)_{3}^{T}, \ldots$ \\
$(2.1)$ & $Z, \lambda_{s}, \lambda_{p}, \lambda_{d}, \ldots\left(\sigma_{1 s}, \sigma_{2 s}, \sigma_{2 p} \ldots\right)$ \\
$(2.2)$ & $\left(k_{1}^{2}\right)_{1},\left(k_{1}^{2}\right)_{2}, \ldots$ \\
\hline
\end{tabular}

In this decimal classification the first figure stands for angular (algebraic) and radial (analytic) branch as before. A ' 1 ' as second figure refers to the target problem dealt with in Section 1, while a ' 2 ' is related to the scattering process. Since a partial wave method is used, the intermediate states $(S L P)_{T}$ of total orbital, spin and parity to be treated in a run must be specified. Storage problems may prevent all the $(S L p)$ 's necessary for convergence being dealt with in one run (see below). Each $(S L p)_{T}$ 
defines one system of coupled channels. The data obtained from (1.1) and (1.2) may be used for one or more isoelectronic ions (2.1), as before, as well as for one or more energies $\left(k_{1}\right)^{2}$ of the exciting electron; the index 1 in the channel energy $k_{i}^{2}$ refers to the ground state.

The three configurations $C_{1}=1 s^{2} 2 s^{2}, C_{2}=1 s^{2} 2 p^{2}, C_{3}=1 s^{2} 2 s 2 p$ as input (1.1) specify a 6-level approximation. For fixed $Z$ and $k_{1}^{2}$, each $(S L p)_{T}$ results in a set of partial collision strengths $\Omega^{(S L p)}\left(i, i^{\prime}\right)$ for transitions between levels $i=\gamma S_{i} L_{i}$. Alternatively reactance matrix elements $R_{\alpha \alpha^{\prime}}$ to $(S L p)_{T}$ may be stored on card output or tape and processed by subroutine SIMMEG, which has been described by Saraph (1970):

$$
\Omega\left(i, i^{\prime}\right)=\sum_{S, L, p} \Omega^{S L p}\left(i, i^{\prime}\right)
$$

SIMMEG constructs the partial collision strength $\Omega^{S L p}$ from $R^{S L p}$. Enough total orbitals $L$ are included when the summation converges. In the 6-level example, $L_{\max }=8$ is sufficient for a $k_{1}^{2}$ not too high above the threshold for exciting the highest level, $2 p^{2}{ }^{1} S$. When including both parities and the two possible $S$, doublets and quartets, 36 intermediate systems are involved. This takes about $8 \mathrm{~min}$ computer time on the IBM 360 . The biggest intermediate states contain $\mathscr{C}=10$ channels. Execution goes about quadratically with $\mathscr{C}$. Thus problems resulting in two dozen coupled channels are easily manageable with this program.

\section{References}

Eissner, W. and Nussbaumer, H.: 1969, J. Phys. B. [2], 2, 1028.

Eissner, W. and Jones, M.: 1970, J. Phys. (Paris), 31 C 4-149.

Jones, M.: 1970, J. Phys. B. [2], 3, 1571.

Saraph, H.: 1970, Computer Phys. Commun. 1, 232. 